\title{
Phase Contrast in Quantum Electron Microscopy
}

\author{
Pieter Kruit $^{1 *}$, Maurice Krielaart ${ }^{1}$ and Yuri van Staaden ${ }^{1}$ \\ 1. Delft University of Technology, Department of Imaging Physics, Delft, The Netherlands. \\ * Corresponding author: p.kruit@tudelft.nl
}

The concept of interaction-free measurements as proposed by Elitzur and Vaidman [3] for photons, should also work with electrons [2]. When built into a transmission microscope [3], this may lead to imaging modes with reduced damage. In the analysis of the image contrast that interaction-free microscopy can create, our tentative conclusion is that we may obtain good contour images of very high amplitude contrast specimen [4]. However, for weak phase objects it is difficult to get an advantage in the interaction-free mode over regular STEM or phase contrast TEM. A multi-pass phase contrast method may be of more value for those specimen [5]. To find out what to expect from the interactionfree, or better "interaction-lean", mode of operation we perform image simulations.

In our scheme, the electron wave is split by an amplitude splitter in a large component (the reference beam) which passes through a hole in the specimen and a small component (the sample beam) that passes through the sample. After the passage, both beams are cycled back to the amplitude splitter and the process is repeated. If the sample has no effect on the beam, the amplitude in the sample beam slowly builds up until it has the full intensity after m cycles. If the sample does have an influence, either on the amplitude or on the phase, the intensity transfer is disturbed (by the quantum Zeno effect), and the intensity stays in the reference beam. Using the model explained in [4], the signals in the reference beam $(\mathrm{R})$ and the sample beam $(\mathrm{S})$ can be calculated as a function of the phase change in the specimen. Figure 1 shows the results for various values of $\mathrm{m}$, here for a transparency $\alpha$ of the sample of 0.7 . Transparency is here the probability that the electron is transmitted without inelastic scattering, so without a collapse of the wave function. The black line (L) represents the inelastic signal which is expected to be proportional to the damage. The horizontal axis can be shifted to an arbitrary value of the phase change by also adding a variable path length change between the reference and the sample beam.

To get an impression of what kind of images QEM would give rise to, we take a fantasy sample of a nano-butterfly of $10 \mathrm{~nm}$ wingspan with a phase pattern in its wings and a constant $\alpha=0,7$. We compensate the phase shift in the outer part of the wings by a path length difference between the reference and the sample beam. In the simulation, we fire a Poisson-distributed random number of electrons into each pixel. After a certain number of cycles, the intensity in the reference beam, respectively the sample beam, is detected. Figure 2 shows the output of a simulated measurement. From left to right, we increase the number of cycles. Because a larger number of cycles allows for a higher resolution at equal damage, we decrease the pixel size in the simulation. However, we keep the average damage approximately constant for all the images. The figure shows that there is only damage in the sample areas where there is signal in the sample beam.

We conclude that it is possible to make images of "iso-phase lines" or "iso-phase areas" in the sample, while only causing damage around these lines or in these areas. We continue our simulations on protein models and try to find out if this image mode would offer an advantage for radiation sensitive materials, with a special interest in cryo microscopy of proteins and high resolution tomography of frozen biological specimen [6]. 
References:

[1] AC Elitzur and L Vaidman, Found. Phys. 23 (1993), p. 987.

[2] W Putnam and M Yanik, Phys. Rev. A 80 (2009), p. 040902.

[3] P Kruit et al., Ultramicroscopy (2016). doi:10.1016/j.ultramic.2016.03.004.

[4] S Thomas et al., Phys. Rev. A 90 (2014), p. 053840.

[5] T Juffmann et al., Scientific Reports 7 (2017), p. 1699. doi:10.1038/s41598-017-01841-x

[6] The authors acknowledge funding from the Gordon and Betty Moore Foundation and the

Netherlands Organization for Scientific Research (NWO).
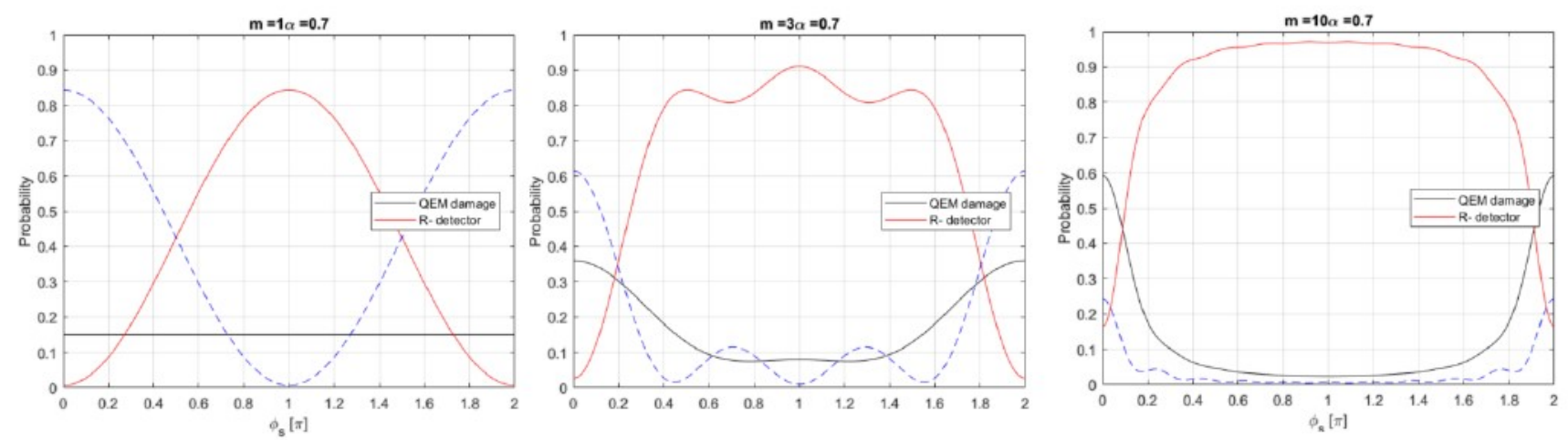

Figure 1. Expected signals as a function of the phase change in the specimen, respectively in the reference beam $(\mathrm{R})$ and the sample beam $(\mathrm{S})$. The black line $(\mathrm{L})$ represents the inelastic signal which is expected to be proportional to the damage.

QEM $m=1$

$\mathrm{R}$

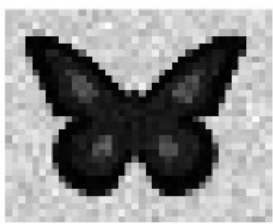

$\mathrm{S}$
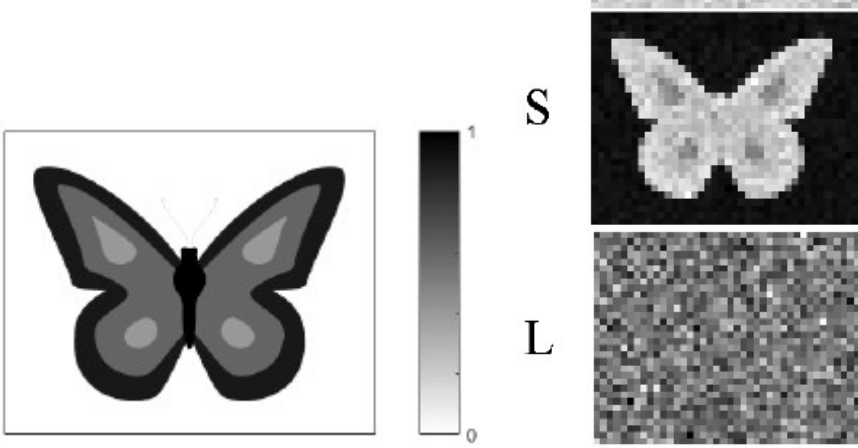

$\mathrm{L}$

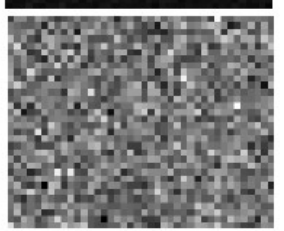

$\mathrm{m}=3$

$\mathrm{m}=10$

$m=30$
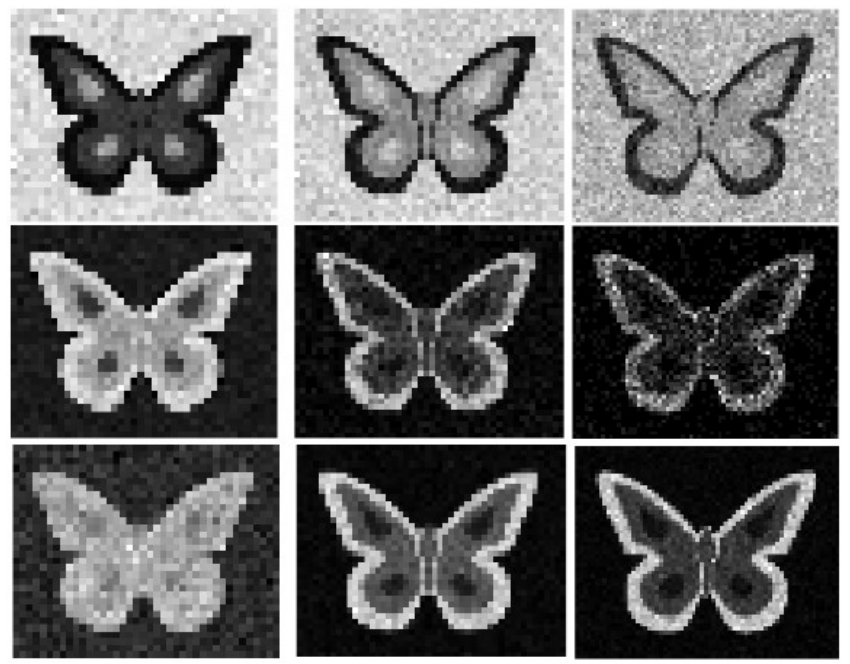

Figure 2. Expected images of a phase object with $\alpha=0.7$ (inset: phase varies from 0 to $\pi$ rad) when imaged respectively with the reference beam $(\mathrm{R})$ or the sample beam $(\mathrm{S})$ for different values of $\mathrm{m}$ (number of cycles in which the reference beam is transferred to the sample beam). The images labeled $\mathrm{L}$ show that there is only damage in the sample areas where there is signal in the sample beam. 